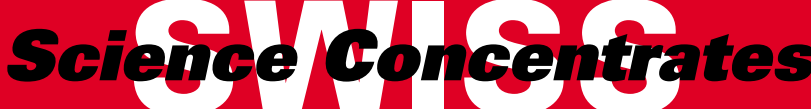

\section{Development of Ruthenium Antitumor Drugs that Overcome Multidrug Resistance Mechanisms}

C. A. Vock, W. H. Ang, C. Scolaro, A. D. Phillips, L. Lagopoulos, L. Juillerat-Jeanneret,* G. Sava, R. Scopelliti, and P. J. Dyson*, J. Med. Chem. 2007, 50, 2166

University Institute of Pathology - CHUV; Institut des Sciences et Ingénierie Chimiques - EPFL; Callerio Foundation; Dipartimento di Scienze Biomediche, Università di Trieste

Multidrug resistance is the appearance of reduced or missing response of microorganisms or cancer cells to applied chemotherapeutic agents and has become a serious problem for the treatment of different diseases. In this paper the authors report the synthesis, characterization and the in vitro cytotoxicity and MDR-reverting properties of organometallic $\eta^{6}-p$-cymene ruthenium(II) complexes bearing modified phenoxazine modulator ligands. Fluorescence microscopy shows that the complexes accumulate much faster in the cell nucleus than the free ligand inducing cell death by inhibition of DNA synthesis.

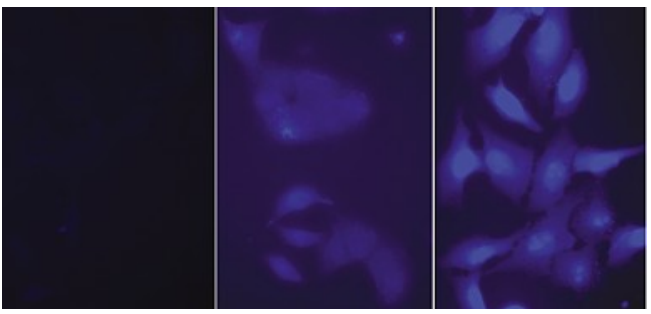

Helical Arrangement of Interstrand Stacked Pyrenes in a DNA Framework

V. L. Malinovskii, F. Samain, and R. Häner*, Angew. Chem., Int. Ed. 2007, 46, 4464

Universität Bern

This article reports the first example of a helical organization within an entirely artificial section of two oligopyrene strands embedded in double-stranded DNA. Interstrand stacking of the pyrene units within the duplex is supported by high thermal stability as well as by UV/vis and fluorescence spectroscopic measurements. The helical organization within the interstrand-stacked oligopyrenes was proven through the observation of exciton-coupled CD signals originating from the pyrene moieties. These findings are important for the design of artificial molecular double-stranded helices for applications in nanotechnology.

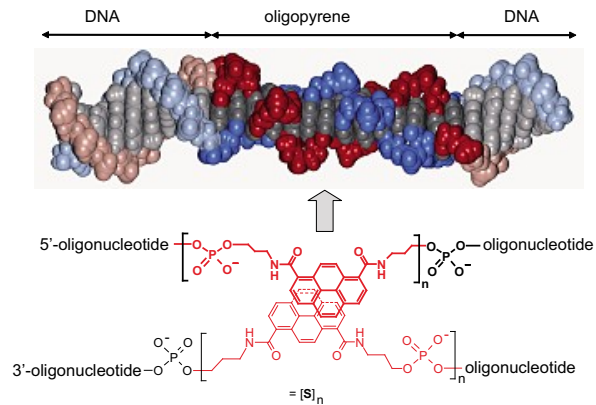

\section{Covalently Functionalized Cobalt Nanoparticles as a Platform for Magnetic Separations in Organic Synthesis}

R. N. Grass, E. K. Athanassiou, and W. J. Stark*, Angew. Chem., Int. Ed. 2007, 46, 4909

Institute for Chemical and Bioengineering, ETHZ

The one-step large scale (>30 $\mathrm{g} \mathrm{h}^{-1}$ ) production of carboncoated magnetic nanobeads is now feasible using reducing flame synthesis. These nanobeads consisting exclusively of metallic cobalt and carbon are highly stable in air at temperatures up to $190{ }^{\circ} \mathrm{C}$ and present excellent magnetic properties. This core-shell material can be covalently functionalized by diazonium chemistry and chloro-, nitro-, and amino-derived particles are readily afforded. These colloidal reagents can now serve as a basis for magnetically functionalized molecules during synthesis, enabling their recovery from reaction mixtures within seconds by application of an external magnetic field.
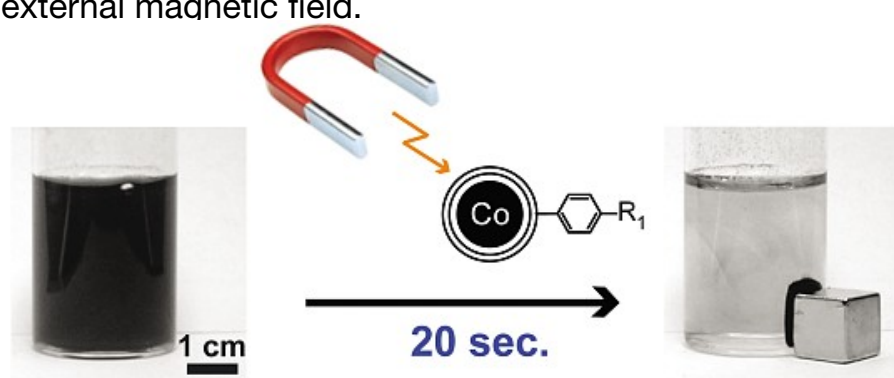

\section{Hierarchical Self-Assembly of One-Dimensional Streptavidin Bundles as a Collagen Mimetic for the Biomineralization of Calcite}

S. Burazerovic, J. Gradinaru, J. Pierron, and T. R. Ward*, Angew. Chem., Int. Ed. 2007, 46, 5510,

University of Neuchâtel

In this article, the versatility of transition-metal connectors in association with proteins bearing tethered ligands to create metal-organic protein frameworks (MOPF) is presented. The introduction of streptavidin (as a linker) between two Fe" (terpyridine-bis-biotin) connectors affords indeed a onedimensional MOPF. In the presence of calcium ions and $\mathrm{CO}_{2}$ vapors, these MOPF aggregates form protein bundles that template the biomineralization of calcite.

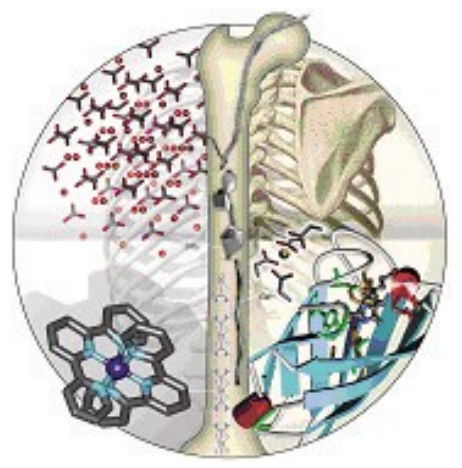

Prepared by M. Austeri; D. Linder; N. Mehanna; R. Novikov; F. Torricelli; J. Lacour

Do you want your article to appear in this SWISS SCIENCE CONCENTRATES highlight?

Please contact concentrates@chimia.ch 